\title{
Inter-center variation in the efficiency of sperm DNA damage reduction following density gradient centrifugation
}

\author{
Carmen López-Fernández ${ }^{1^{*}}$, Stephen D. Johnston ${ }^{2}$, Altea Gosálbez ${ }^{1}$, Jose Luís Fernández ${ }^{3}$, \\ Juan G. Álvarez ${ }^{4}$, Jaime Gosálvez ${ }^{1}$ \\ ${ }^{1}$ Department of Biology, Genetics Unit, University Autónoma of Madrid, Madrid, Spain; \\ *Corresponding Author: kcchou@gordonlifescience.org \\ ${ }^{2}$ School of Agriculture and Food Science, The University of Queensland, Gatton, Australia \\ ${ }^{3}$ Laboratorio de Genética Molecular y Radiobiología, Centro Oncológico de Galicia, A Coruña, Spain \\ ${ }^{4}$ Centro ANDROGEN, A Coruña, Spain
}

Received 23 May 2013; revised 24 June 2013; accepted 2 July 2013

Copyright (C) 2013 Carmen López-Fernández et al. This is an open access article distributed under the Creative Commons Attribution License, which permits unrestricted use, distribution, and reproduction in any medium, provided the original work is properly cited.

\section{ABSTRACT}

This was a prospective multicenter study aiming at comparing the efficiency of sperm selection by density gradient centrifugation (DGC) in reducing sperm DNA fragmentation (SDF) in different ART centers. The study was designed using 290 semen samples collected from 10 different ART centers performing artificial insemination, in vitro fertilization and blind assessment of SDF at the University facilities. The results showed that while there was a significant reduction in the SDF levels in sperm isolated from the gradient centrifuged pellet (DGC) compared to neat semen samples (NSS), there was also significant inter-center variability in the efficiency to reduce SDF values by DGC $\mathbf{( 7 8 . 5 \%}$ to 29.2\%). Surprisingly, for some patients, the level of SDF actually increased following sperm selection. The main conclusions derived from this study were that 1 ) isolation of sperm from the gradient pellet by DGC must be performed using validated, optimized protocols; 2) routine comparison of SDF values in NSS semen and in processed sperm after DGC or swim-up must be recommended as part of the internal quality control (QC) of ART laboratories to test the efficacy of sperm processing; and 3) SDF values in processed spermatozoa should be obtained to compare with the pregnancy rate when insemination or fertilization is about to be performed, otherwise, attempts to predict pregnancy outcome from SDF could be biased or are essentially meaningless.
Keywords: Assisted Reproductive Technology; Fertility; Sperm DNA Fragmentation; Male Factor; Semen Processing

\section{INTRODUCTION}

Predicting the success rate of human pregnancy using estimates of sperm DNA damage to date has been a very controversial issue, and that is no doubt further complicated by a plethora and permutation of techniques and procedures that vary in competency and delivery from laboratory to laboratory, all of which result in a fundamental lack of standardization. Figure 1 illustrates some of the potential factors impacting on the success of SDF as a predictive measure and its relative interconnectedness with other assisted reproduction parameters. These variables include 1) the type of fertilization technique

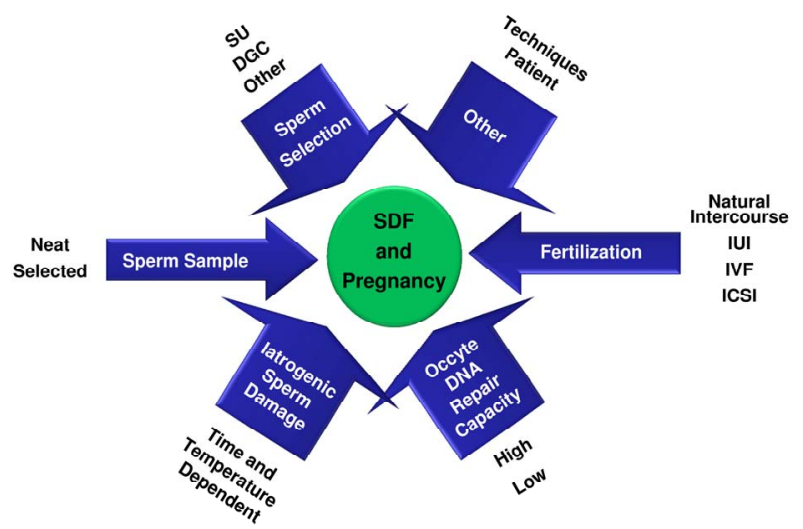

Figure 1. Factors affecting the reliability of sperm DNA fragmentation as a predictor of pregnancy outcome. 
(e.g. natural intercourse, intrauterine insemination [IUI], in vitro fertilization [IVF] and intra cytoplasmic sperm injection (ICSI) [1-11]); 2) the use of neat or selected spermatozoa $[4,12]$; 3) the specific techniques used for sperm selection such as swim-up, density gradient centrifugation (DGC) whether used alone or in combination with intracytoplasmic morphologically-selected sperm injection (IMSI), Physiological ICSI (PICSI) or Magnetic activated cell sorting (MACS) [13-16]; 4) iatrogenic induced sperm damage during sperm ex vivo handling $[17,18]$; 5) the type of technique used to assess DNA damage [19]; and 6) the quality of the donor oocyte [20-22].

Given the wide range of variables that contribute to the success of an ART outcome, it is therefore not surprising that this variability is also reflected in the ability of sperm DNA fragmentation (SDF) to predict pregnancy outcome. This concept is well illustrated if we consider just one of the variables in Figure 1, such as sperm selection. In order to improve the predictive power of SDF it is important that we account and describe the precise conditions used when conducting our sperm selection procedure; these issues might include how long the sperm was stored after ejaculation and prior to sperm selection, what specific type of sperm selection procedure was employed, for how long and under what conditions was the sperm sample stored or incubated prior to fertilization and what was the quality of the donor oocytes to be used? In fact, it is logical to assume that the quality of the oocyte contributes a minimum of $50 \%$ to predictive nature of sperm DNA for pregnancy outcome.

We would, therefore, contend that if SDF is to be used as a predictive measure of pregnancy success, then there needs be close attention given to standardizing the specific processes and conditions of ART that are used in the different laboratories. Even when such standardization is accounted for, there is still the potential for innate variability in the efficiency of SDF reduction usually observed after sperm selection among different individuals processed within the same laboratory [23]; this phenomenon is likely to be related to inherent differences associated with the ejaculate of each patient, to the technical competencies of the respective andrologist's ability to process the sperm sample or a synergistic combination of both. In the current study, we explore this general idea by investigating the relative efficiency of standardized density gradient centrifugation protocols (DGC), as routinely used in ART clinics to improve sperm DNA quality.

\section{MATERIAL AND METHODS}

A total of 290 semen samples from 10 different Spanish ART Centers performing artificial insemination and in vitro fertilization were included in this study. All pa- tients consented to their participation according Spanish legislation and confidentiality and following adherence to Spanish government ethical standards and the internal ethics committee of the participating clinic. The study used a double blind strategy for sperm collection and for sperm analysis. All the semen samples were processed in each clinic as per "in house" standard protocols. Two semen samples per ejaculate were prepared for SDF analysis; 1) Neat semen samples (NSS) were cryopreserved using standard procedures following immediate liquefaction of the ejaculate; and 2) Selected semen samples were processed following liquefaction and density gradient centrifugation (DGC) at the time equivalent to that of fertilization and then cryopreserved as per the NSS to be analyzed at a different facility. Each sample was processed using the standard methodologies for DGC as routinely used in each clinic; consequently in our analysis of results we made no attempt to standardize reagents or methodologies used for DGC in each center or methodologies used for cryopreservation. Rather, we focussed our attention on the final net result of the efficiency to reduce the level of SDF with respect to the NEAT recovered in each clinic.

Semen samples were collected from case studies of patients and the decision for patient inclusion was taken by an andrologist without coordination or reference to technicians processing the samples. In order to be included in the study, the neat semen samples were required to have a sperm concentration of greater than $20 \mathrm{x}$ 106 per $\mathrm{mL}$ and a sperm motility of $>50 \%$. Patients with varicocele, teratozoospermia, oligozoospermia, leukocytospermia were excluded from the study. Cryopreserved samples from each center were sent to the Unit of Genetics at the University Autónoma de Madrid, where all the samples were assessed for sperm DNA damage (Halosperm G2-Halotech, Madrid, Spain) by the same technician.

To avoid iatrogenic DNA damage, SDF was immediately assessed after thawing. NSS and DGC samples were diluted to achieve a concentration of $10 \times 106$ per $\mathrm{ml}$ in Modified Ham's F-10 Basal Medium-HEPES (Irvine Scientific, CA, USA) and assessed for the level of SDF. After comparing the values of SDF using Halosperm G2 (Halotech, Madrid, Spain) obtained for NSS and DGC samples, the efficiency in sperm DNA damage reduction, defined as the e-value (percent reduction of SDF when NSS and DGC samples are compared) was calculated.

SPSS v.15.0 for Windows (SPSS Inc., Chicago, IL, USA) was used for all statistical analysis. Box-andwhisker plots were used to show the distribution of the assessed variable among different ART clinics. Differences in SDF values among different centers were determined using a Kruskal-Wallis test. The Wilcoxon signed-rank test for a non-parametric analysis was used to 
compare two related samples. The Fisher's Least Significant Difference test was used to compare and arrange differences in the efficiency to reduce SDF using group means. Correlation analysis was performed using a nonparametric Pearson test.

\section{RESULTS}

Table 1 shows the mean and standard deviation of NSS and DGC samples and $e$-value for each ART center. Figure 2 summarizes the descriptive statistics of SDF values across the 10 ART centers examined in this study. There were significant differences in mean SDF values of both NSS (Kruskal-Wallis; $\chi^{2}=9.7 ; \mathrm{P}=0.00$ ) and DGC (Kruskal-Wallis; $\chi^{2}=78.1 ; \mathrm{P}=0.00$ ) when different centers were compared (Figures 2(a) and (b)). When data for all ART centers was pooled and the efficiency

Table 1. Mean $\pm(\mathrm{SD})$ values for sperm DNA fragmentation (SDF) of neat semen samples (NSS) and density gradient centrifuged (DGC) samples obtained in 10 different ART centers. The efficiency for SDF reduction- $e$-value-was expressed as the percentage of variation obtained for SDF after comparing NSS and DGC.

\begin{tabular}{ccccc}
\hline Control & $\mathbf{n}$ & NSS & DCG & Reduction in SDF e-value \\
\hline 1 & 13 & $32.2 \pm 3.6^{\mathrm{A}}$ & $17.3 \pm 3.2^{\mathrm{B}}$ & $49.6 \pm 9.6$ \\
2 & 21 & $21.8 \pm 2.8^{\mathrm{A}}$ & $5.2 \pm 2.5^{\mathrm{B}}$ & $76.0 \pm 7.5$ \\
3 & 19 & $26.8 \pm 3.0^{\mathrm{A}}$ & $9.7 \pm 2.6^{\mathrm{B}}$ & $67.1 \pm 7.9$ \\
4 & 30 & $43.9 \pm 2.4^{\mathrm{A}}$ & $28.8 \pm 2.1^{\mathrm{B}}$ & $34.1 \pm 6.3$ \\
5 & 18 & $37.1 \pm 3.1^{\mathrm{A}}$ & $9.7 \pm 2.7^{\mathrm{B}}$ & $71.6 \pm 8.1$ \\
6 & 45 & $26.3 \pm 1.9^{\mathrm{A}}$ & $14.8 \pm 1.7^{\mathrm{B}}$ & $36.2 \pm 5.1$ \\
7 & 27 & $25.5 \pm 2.5^{\mathrm{A}}$ & $6.8 \pm 2.2^{\mathrm{B}}$ & $78.5 \pm 6.6$ \\
8 & 40 & $18.5 \pm 2.0^{\mathrm{A}}$ & $12.7 \pm 1.8^{\mathrm{B}}$ & $29.1 \pm 5.4$ \\
9 & 33 & $30.0 \pm 2.3^{\mathrm{A}}$ & $17.1 \pm 2.0^{\mathrm{B}}$ & $44.8 \pm 6.0$ \\
10 & 44 & $27.1 \pm 1.9^{\mathrm{A}}$ & $11.3 \pm 1.7^{\mathrm{B}}$ & $60.6 \pm 5.2$ \\
Pooled & 290 & $28.9 \pm 7.4^{\mathrm{A}}$ & $13.3 \pm 6.8^{\mathrm{B}}$ & $54.8 \pm 18.4$ \\
\hline
\end{tabular}
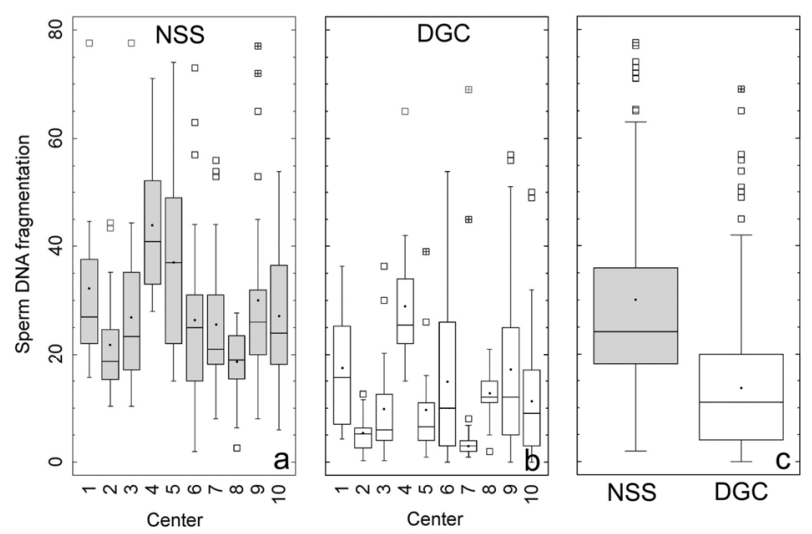

Figure 2. Box Whisker plot showing the distribution of sperm DNA fragmentation (SDF) in (a) neat semen samples (NSS); and (b) density gradient processed (DGC) semen samples from different Spanish ART centers (1 - 10); (c) Pooled mean in NSS and DGC SDF values for all centers. for SDF calculated, there was a $54.8 \%$ reduction in SDF $(e$-value $=54.8 \%)$ following DGC (Table 1 and Figure 2(c)). There were significant differences in the efficiency for SDF reduction between the different centers (Kruskal-Wallis; $\left.\chi^{2}=87.1 ; \mathrm{P}=0.00\right)$.

ART centers were then ranked according to their respective $e$-value (Table 2). Center 7 was very successful in selecting high-quality sperm from the DGC pellet with an overall reduction efficiency of approximately $80 \%$. At the other end of the spectrum, Center 6 had a reduction efficiency of only $29 \%$. Following analysis using the Fisher's Least Significant Difference test to compare group means, significant differences in the efficiency to reduce SDF were obtained, whereby it was possible to statistically differentiate 5 different levels of centers based on their efficiency to reduce SDF (Table 2). This analysis essentially revealed that 5 clinics were efficient at reducing SDF, ranging from an e-value of $60 \%$ to $80 \%$, while the remainder presented an e-value ranging between $30 \%$ and $50 \%$.

Although the raw data are not presented, $8 \%$ of individual patient cases across all the centers actually resulted in an increase of SDF following selection by DGC when compared to the corresponding neat semen samples. The lowest efficiency was observed again in Center 6 , where $20 \%$ ( $9 / 45$ cases) of the samples subjected to DGC yielded higher levels of DNA damage in the DGC pellet compared to the NSS.

As differences were found in the SDF of the neat semen samples from the different clinics, we examined by means of a correlation analysis, whether the e-value, was dependent on the initial SDF of the neat semen sample. This analysis revealed no significant correlation between these variables (Pearson $=-0.01 ; \mathrm{n}=290$; Figure 3(a)); this lack of correlation was also upheld when the data for

Table 2. Efficiency of sperm DNA fragmentation (SDF) reducetion in each center and ranking efficiency per center. Five different groups (ranked and groups from 1 to 5 in the right column) were predicted according to the Fisher's Least Significant Difference test; e-value represents SDF reduction after comparing SDF values obtained in NSS and DGC.

\begin{tabular}{ccc}
\hline Centre & \% SDF Reduction (e-value) Mean & Group Rank \\
\hline 7 & 78.5 & 1 \\
2 & 76.0 & 21 \\
5 & 71.6 & 321 \\
3 & 67.1 & 321 \\
10 & 60.6 & 43 \\
1 & 49.6 & 54 \\
9 & 44.8 & 5 \\
6 & 36.2 & 5 \\
4 & 34.1 & 5 \\
8 & 29.1 & 5 \\
\hline
\end{tabular}



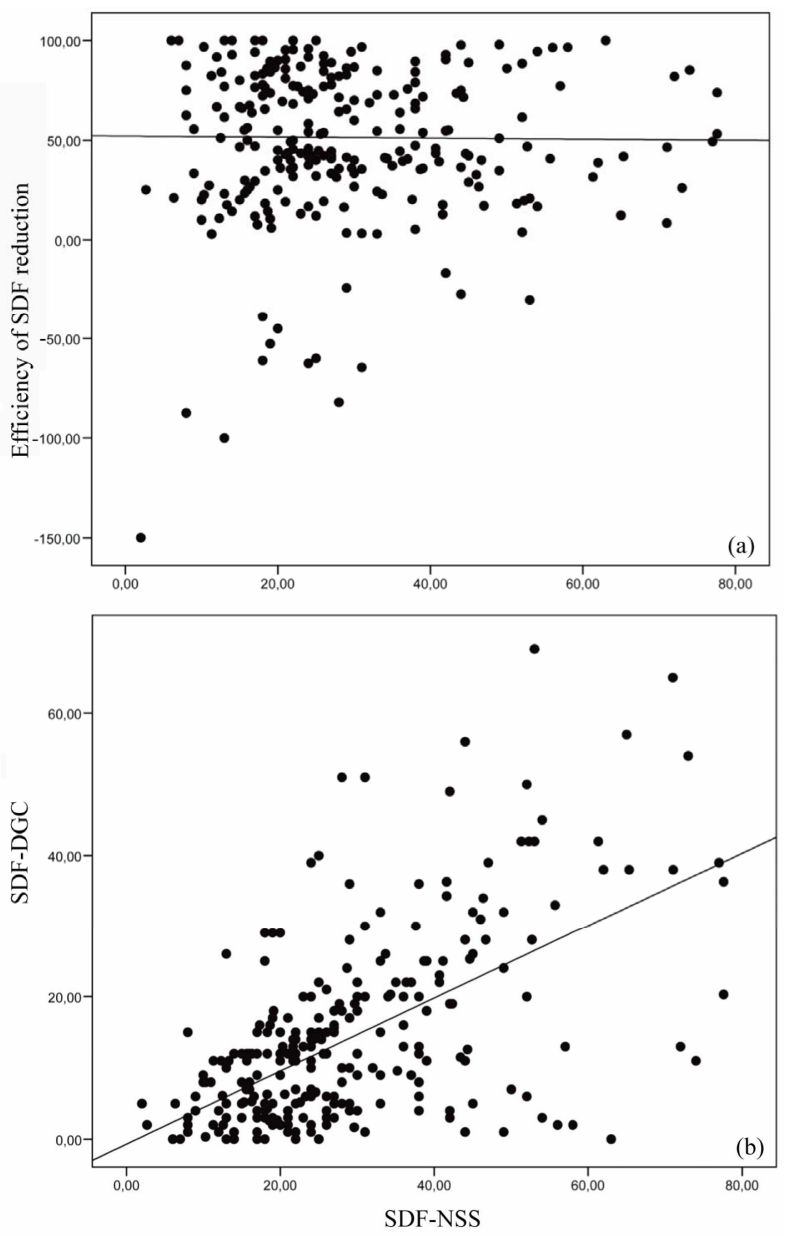

Figure 3. Graphic representation of the correlation between (a) $\%$ sperm DNA fragmentation (SDF) of the neat semen sample (NSS) and the efficiency of sperm DNA fragmentation (SDF) reduction and (b) SDF of NSS plotted against SDF in DCG samples.

each center was plotted separately. Collectively, these findings appear to indicate that the SDF value in the NSS was not influencing the efficiency of the selection procedure in reducing SDF in the DGC pellet.

Despite the lack of correlation between the level of SDF in the NSS and the efficiency for SDF reduction after DGC, there was still a significant correlation, using Pearson's test (Pearson $=0.58 ; \mathrm{P}<0.01 ; \mathrm{n}=290$ ), between SDF observed in the NSS and SDF obtained in DGC samples (Figure 3(b)), i.e. generally low/high levels of SDF in the NS give rise to low/high SDF following the DGC procedure.

\section{DISCUSSION}

Two main conclusions emerge of this study 1) in general, there was a significant reduction in the levels of SDF in sperm isolated in the gradient pellet following DGC; and 2) there was significant inter-center variability in the efficiency to select DNA intact spermatozoa from the gradient pellet following DGC.

The reported reduction in sperm DNA damage after sperm selection is not a novel observation as this effect has been previously reported in other studies [14,24-26]. Rather, the uniqueness of this study was that it examined the efficiency to achieve such a reduction across multiple centers; it was clear from our analysis that the level of SDF reduction was significantly different across the ART centers. It is highly likely that this variability in the efficiency of sperm recovery with low levels of DNA damage will have a significant impact on the pregnancy rate achieved in each of the respective centers, since the probability of selecting one sperm containing a damaged DNA molecule is expected to be higher if the efficiency to remove the damage is low. Our results suggest that it would be prudent for some centers to develop better methods of quality control for the sperm selection procedures.

In the present study and according to the grouping criteria obtained from the Fisher's Least Significant Difference test (Table 2), Centers 7, 2, 5 and 3 (as ranked in Table 2) showed the most efficiency in terms of relative sperm DNA quality improvement. The $e$-value for SDF reduction in this group ranged from $68 \%-78 \%$ while those in less efficient group (Centers 8, 4, 6, 9 and 1) ranged from $30 \%$ to $50 \%$. We perceive three plausible explanations for these observations. Firstly, we suggest that the inter-center differences are related to the possible differences in the design and performance of the DGC protocol, which may be related to centrifugation speed, centrifugation time and the ability to aspirate a clean pellet. We also suggest that the skill associated with the isolation of the different density gradient layers may also be a significant factor contributing to this variability, because layer mixing and contamination may occur during aspiration of fractions 1 and 2, where the highest levels of immature spermatozoa and the higher levels of affected sperm are typically found [27]. This is likely to be the most parsimonious explanation and would possibly account for those cases where the level of SDF after DGC was actually higher than that found in the NSS.

Secondly, even though there were relatively strict inclusion parameters set for this study, the quality of the individual semen samples e.g., percentage of ROS-producing immature spermatozoa [28,29] is also likely to be an additional factor contributing to the observed inter-center variability, especially since the initial SDF values obtained in neat semen would be significantly magnified following processing and this could be easily linked to an accumulative presence of sperm damage at different levels [23,30]. Either independently or synergistically, these factors could act to produce subtle differences that influence sperm DNA survival. 
The other potential explanation for the observed variability between clinics with respect to the efficacy in the reduction of spermatozoa with DNA damage after DGC could be the often overlooked negative impact of iatrogenic damage prior to, during and following sperm selection. Sperm DNA damage tends to increase after ejaculation and differences in the sperm DNA longevity exists among different individuals. This phenomenon has been previously been described [31,32] and appears to be related to the lag time between sperm selection and DNA damage analysis. The dilemma as we see it is that the high efficiency of SDF reduction in some centers (close to $80 \%$ ) may in fact only be compensating for the iatrogenic DNA damage inherent to the DGC protocol, rendering the DGC a potentially redundant process. When the level of SDF after the DGC protocol is higher than that of the NSS, as it happens in some of the cases reported in this study or published elsewhere [23], the reality is that we may be wasting our time and in fact, in some cases actually reducing the chance of successful pregnancy by increasing the level of SDF when exposing sperm to selection procedures.

\section{CONCLUSIONS}

We derive three main "take-home" messages from this study: 1) isolation of sperm from the gradient pellet by DGC must be performed using validated, optimized protocols; this should reduce the observed inter-center variation, not only in SDF values but also for other seminal parameters; 2) routine comparison of SDF values in NSS and DGC or swim-up should be recommended as part of the internal quality control of the ART laboratory to test the safety and efficacy of sperm processing; and 3) SDF values in processed spermatozoa should be obtained to compare with the pregnancy rate when insemination or fertilization is about to be performed, otherwise, attempts to predict pregnancy outcome from SDF could be essentially confusing if not meaningless.

\section{ACKNOWLEDGEMENTS}

The author(s) declared no potential conflicts of interest with respect to the research, authorship, and/or publication of this article. This research was supported with public funding (Spanish Ministry of Science and Technology (MCYT: BFU2010-16738).

\section{REFERENCES}

[1] Lopes, S., Sun, J.G., Jurisicova, A., Meriano, J. and Casper, R.F. (1998) Sperm deoxyribonucleic acid fragmentation is increased in poor-quality semen samples and correlates with failed fertilization in intracytoplasmic sperm injection. Fertility and Sterility, 69, 528-532.

doi:10.1016/S0015-0282(97)00536-0
[2] Evenson, D.P., Jost, L.K., Marshal, D., Zinaman, M.J., Clegg, E., Purvis, K., De Angelis, P. et al. (1999) Utility of the sperm chromatin structure assay as a diagnostic and prognostic tool in the human fertility clinic. Human Reproduction, 14, 1039-1049.

doi:10.1093/humrep/14.4.1039

[3] Spano, M., Bonde, J.P., Hjollund, H.I., Kolstad, H.A., Cordelli, E. and Leter, G. (2000) Sperm chromatin damage impairs human fertility. The Danish first pregnancy planner study team. Fertility and Sterility, 73, 43-50.

[4] Bungum, M., Humaidan, P., Spano, M., Jepson, K., Bungum, L. and Giwercman, A. (2004) The predictive value of sperm chromatin structure assay (SCSA) parameters for the outcome of intrauterine insemination, IVF and ICSI. Human Reproduction, 19, 1401-1408. doi:10.1093/humrep/deh280

[5] Collins, J.A., Barnhart, K.T. and Schlegel, P.N. (2008) Do sperm DNA integrity tests predict pregnancy with in vitro fertilization? Fertility and Sterility, 89, 823-831. doi:10.1016/j.fertnstert.2007.04.055

[6] Bakos, H.W., Thompson, J.G., Feil, D. and Lane, M. (2008) Sperm DNA damage is associated with assisted reproductive technology pregnancy. International Journal of Andrology, 31, 518-526. doi:10.1111/j.1365-2605.2007.00803.x

[7] Castilla, J.A, Zamora, S., Gonzalvo, M.C., Luna Del Castillo, J.D., Roldan-Nofunentes, J.A., Clavero, A. et al. (2010) Sperm chromatin structure assay and classical semen parameters: Systematic review. Reproductive Biomedicine Online, 20, 114-124. doi:10.1016/j.rbmo.2009.10.024

[8] Gandini, L., Lombardo, F., Paoli, D., Caruso, F. Eleuteri, P., Criminna, R. et al. (2004) Full-term pregnancies achieved with ICSI despite high levels of sperm chromatin damage. Human Reproduction, 19, 1409-1417. doi:10.1093/humrep/deh233

[9] Li, Z., Wang, L., Cai, J. and Huang, H. (2006). Correlation of sperm DNA damage with IVF and ICSI outcomes: A systematic review and meta-analysis. Journal of Assisted Reproduction and Genetics, 23, 367-376. doi:10.1007/s10815-006-9066-9

[10] Virro M.R., Larson-Cook, K.L. and Evenson, D.P. (2005) Sperm chromatin structure assay (SCSA) parameters are related to fertilization, blastocyst development, and ongoing pregnancy in vitro fertilization and intracytoplamic sperm injection cycles. Fertility and Sterility, 81, 12891295. doi:10.1016/j.fertnstert.2003.09.063

[11] Simon, L., Castillo, J., Oliva, R. and Lewis, S.E. (2011) Relationships between human sperm protamines, DNA damage and assisted reproduction outcomes. Reproductive Biomedicine Online, 23, 724-734. doi:10.1016/j.rbmo.2011.08.010

[12] Santiso, R., Tamayo, M., Gosálvez, J., Meseguer, M. Garrido, N and Fernandez, J.L. (2010) Swim-up procedure selects spermatozoa with longer telomere length. Mutation Research, 688, 88-90. doi:10.1016/j.mrfmmm.2010.03.003

[13] Tampaichitr, N., Agulnik, A., Seibel, M. and Taymor, M. (1988) Comparison of the in vitro fertilization rate by 
human sperm capacitated by multiple-tube swim-up and Percoll gradient centrifugation. Journal of in Vitro Fertilization and Embryo Transfer, 5, 119-122. doi:10.1007/BF01131172

[14] Zini, A., Finellia, A., Phanga, D. and Jarvi, K. (2000) Influence of semen processing technique on human sperm DNA integrity. Urology, 56, 1081-1084. doi:10.1016/S0090-4295(00)00770-6

[15] Bungum, M., Spano, M., Humaidan, P., Eleteri, P., Rescia, M. and Giwercman, A. (2008) Sperm chromatin structure assay parameters measured after density gradient centrifugation are not predictive for the outcome of ART. Human Reproduction, 23, 4-10. doi:10.1093/humrep/dem353

[16] Enciso, M., Iglesias, M., Galán, I., Sarassa, J. Gosalvez, A. and Gosalvez, J. (2011) The ability of sperm selection techniques to remove single- or double-strand DNA damage. Asian Journal of Andrology, 13, 764-768. doi:10.1038/aja.2011.46

[17] Mortimer, D. (1991) Sperm preparation techniques and iatrogenic failures of in vitro fertilization. Human Reproduction, 6, 173-176.

[18] Gosálvez, J., López-Fernández, C., Fernández, J.L., Gourand, A. and Holt, W.V. (2011) Relationships between the dynamics of iatrogenic DNA damage and genomic design in mammalian spermatozoa from eleven species. Molecular Reproduction and Developmen, 78, 951-961. doi:10.1002/mrd.21394

[19] Gosálvez J., López-Fernández, C. and Fernández, J.L. (2011) Sperm Chromatin Dispersion (SCD) test: Technical aspects and clinical applications. In: Zini, A., Agarwal, A., Eds., Sperm DNA damage: Biological and clinical applications in male infertility and assisted reproduction. Springer Verlag, New York, 151-170.

[20] Menezo, Jr. Y., Russo, G.L., Tosti, E., Moutassim, S. and Benkhalifa, M. (2007) Expression profile of genes coding for DNA repair in human oocytes using pangenomic microarrays, with a special focus on ROS linked decays. Journal of Assisted Reproduction and Genetics, 24, 513520. doi:10.1007/s10815-007-9167-0

[21] Meseguer, M., Santiso, R., Garrido, N., Garcia-Herrero, S., Remohi, J. and Fernandez, J.L. (2011) Effect of sperm DNA fragmentation on pregnancy outcome depends on oocyte quality. Fertility and Sterility, 95, 124-128. doi:10.1016/j.fertnstert.2010.05.055

[22] Nuñez-Calonge, R., Caballero, P., López-Fernández, C., Guijarro, J.A., Fernandez, J.L., Johnston, S. and Gosalvez, J. (2012) An improved experimental model for understanding the impact of sperm DNA fragmentation on human pregnancy following ICSI. Reproductive Science, 19, 1163-1168. doi:10.1177/1933719112459238

[23] Gosálvez, J., De La Torre, J., López-Fernández, C., Perez-Gutierrez, L., Ortega, L., Caballero, P. and Nunez, R.
(2010) DNA fragmentation dynamics in fresh versus frozen thawed 1 plus gradient-isolated human spermatozoa. Systems Biology in Reproductive Medicine, 56, 27-36. doi:10.3109/19396360903515430

[24] Parmegiani, L., Cognigni, G.E., Bernardi, S., Troilo, E., Ciampaglia, W. and Filicori, M. (2010) "Physiologic ICSI": Hyaluronic acid (HA) favors selection of spermatozoa without DNA fragmentation and with normal nucleus, resulting in improvement of embryo quality. Fertility and Sterility, 93, 598-604. doi:10.1016/j.fertnstert.2009.03.033

[25] Tavalaee, M., Deemeh, M.R., Arbabian, M. and NasrEsfahani, M.H. (2012) Density gradient centrifugation before or after magnetic-activated cell sorting: Which technique is more useful for clinical sperm selection? Journal of Assisted Reproduction and Genetics, 29, 31-38. doi:10.1007/s10815-011-9686-6

[26] Ebner, T., Shebl, O., Moser, M., Arzt, W. and Tews, G. (2011) Easy sperm processing technique allowing exclusive accumulation and later usage of DNA-strand break-free spermatozoa. Reproductive Biomedicine Online, 22, 37-43. doi:10.1016/j.rbmo.2010.09.004

[27] Ollero, M., Gil-Guzman, E., Lopez, M.C., Sharma, R.K., Agarwal, A., Larson, K. et al. (2001) Characterization of subset of human spermatozoa at different stages of maturation: Implications in the diagnosis and treatment of male infertility. Human Reproduction, 16, 1912-1921. doi:10.1093/humrep/16.9.1912

[28] Agarwal, A., Makker, K. and Sharma, R. (2008) Clinical relevance of oxidative stress in male factor infertility: An update. American Journal Reproductive Immunology, 59, 2-11. doi:10.1111/j.1600-0897.2007.00559.x

[29] Aitken, R.J. and De Iuliis, G.N. (2010) On the possible origins of DNA damage in human spermatozoa. Molecular Human Reproduction, 16, 3-13. doi:10.1093/molehr/gap059

[30] López-Fernández, C., Johnston, S.D., Fernández, J.L., Wilson, R.J. and Gosalvez, J. (2010) Fragmentation dynamics of frozen-thawed ram sperm DNA is modulated by sperm concentration. Theriogenology, 74, 1362-1370. doi:10.1016/j.theriogenology.2010.06.006

[31] Gosálvez, J., Cortés-Gutierrez, E., López-Fernádez C., Fernandez, J.L., Caballero, P. and Nunez, R. (2009) Sperm DNA fragmentation dynamics in fertile donors. Fertility and Sterility, 92, 170-173. doi:10.1016/j.fertnstert.2008.05.068

[32] Calamera, J.C., Buffone, M.G., Doncel, G.F., Brugo-Olmedo, S., De Vincentiis, S., Calamera, M.M. et al. (2010) Effect of thawing temperature on the motility recovery of cryopreserved human spermatozoa. Fertility and Sterility, 93, 789-794. doi:10.1016/j.fertnstert.2008.10.021 